\title{
Three-dimensional topology of turbulent premixed flame interaction
}

\author{
R.A.C. Griffiths ${ }^{* 2,3}$, J.H. Chen ${ }^{1}$, H. Kolla ${ }^{1}$, R.S. Cant $^{2}$, W. Kollmann ${ }^{3}$ \\ ${ }^{1}$ CRF, Sandia National Laboratories, Livermore, CA 94550,USA \\ ${ }^{2}$ Department of Engineering, University of Cambridge, U.K. \\ ${ }^{3}$ MAE Dept, University of California Davis, CA 95616, USA \\ Email: racg3@cam.ac.uk
}

\begin{abstract}
The topology of turbulent premixed flames is analysed using data from Direct Numerical Simulation (DNS), with emphasis on the statistical geometry of flame-flame interaction. A general method for obtaining the critical points of line, surface and volume fields is outlined, and the method is applied to isosurfaces of reaction progress variable in a DNS configuration involving a pair of freely-propagating hydrogen-air flames in a field of intense shear-generated turbulence. A complete set of possible flame-interaction topologies is derived using the eigenvalues of the scalar Hessian, and the topologies are parametrised using a pair of shape factors. The frequency of occurrence of each type of topology is evaluated from the DNS dataset for two different Damköhler numbers. Different types of flame-interaction topology are found to be favoured in various regions of the turbulent flame, and the physical significance of each interaction is discussed.
\end{abstract}

Keywords: Turbulent premixed flame; DNS; flame structure; flame topology

\section{Introduction}

Direct Numerical Simulation (DNS) results for turbulent flames are becoming available for increasing Reynolds numbers [1, 2, 3], thus making it possible to carry out a detailed analysis of 
the structural properties of the flame. The dissipative range of length scales is of particular interest since combustion reactions take place at small length and time scales. This emphasises the need for highly-resolved DNS data containing the greatest possible range of scales. The structure of prime importance in combustion at high Damköhler number is the flame front itself. It can be located as an embedded surface defined as a chosen level surface of a suitable scalar field. In premixed combustion the usual scalar of choice is the reaction progress variable. The geometry and local topology of the flame surface is central to turbulent premixed combustion modelling using the flamelet concept, and especially in modelling using the Flame Surface Density (FSD) equation or the G-equation approach $[4,5]$. Processes related to the local flame topology, such as cusp formation or pocket burnout, can have a significant effect on the overall balance of flame area production and destruction and hence affect the overall burning rate. Flame topology is also highly relevant to the occurrence of intermittent events such as local extinction [6]. A good fundamental understanding of these phenomena is essential if they are to be captured accurately in modelling.

The statistical geometry of turbulent premixed flames has been addressed in previous work which has highlighted the importance of flame curvature in determining propagation behaviour $[7,8]$. The specific issue of pocket formation through flame pinchoff has been analysed in detail using DNS data for the two-dimensional case $[9,10]$. Topological aspects of scalar field structure have been analysed in non-reacting turbulent flows [11, 12], and aspects of flame structure have been addressed [13]. Recently, DNS data has been used to elucidate the topology of flame structure in MILD combustion [14]. The present work is aimed at analysing the topology of freely-propagating turbulent premixed flames using DNS data in three dimensions. The general principles will be outlined, and the specific DNS datasets will be described. The set of possible topologies for flame-flame interaction in three dimensions will be presented, the frequency of occurrence of each type of interaction will be evaluated, and some observations will be made concerning the local flame propagation behaviour. 


\section{Spatial/Eulerian structures}

Spatial structures are defined as measurable subsets of the flow domain, fully specified by the relevant physical and chemical properties, and specified in the spatial/Eulerian description [15]. The notion of measure is taken as a generalisation of properties such as length, area, volume etc. [16]. The most important and elementary properties of the subsets are the measure denoted by $V_{0}$ and its dimension, the area of the boundary $V_{1}$ and its dimension, and the mean and Gaussian curvature integrated over the bounding surface, denoted by $V_{2}$ and $V_{3}$ respectively. The $V_{i}$ are called the Minkowski functionals [17]. The usual definition of dimension as the number of basis vectors (coordinates) required to specify any vector is not sufficient and a more general point of view must be taken $[18,19]$.

\subsection{Line type structures}

Subsets of the flow field $\mathcal{D} \subset R^{3}$ with Hausdorff dimension $d_{H} \leq 1$ are called line type structures. The vector fields defined on $\mathcal{D}$ such as velocity and vorticity generate tangential vectorlines according to

$$
\frac{d x_{\alpha}}{d s}=v_{\alpha}(\mathbf{x}(s), t), \quad \alpha=1,2,3
$$

with an arbitrary initial condition $\mathbf{x}(0)=\mathbf{x}_{0} \in \mathcal{D}$ where $s$ denotes the arclength measured along the vectorline. Time $t$ is constant in the spatial description, and hence the system of ordinary differential equations is always autonomous with respect to arclength $s$. For a finite set of initial conditions $\mathbf{x}^{i}, i=1, \cdots, N$, the vectorlines through the set can be analyzed with the aid of algebraic topology including braids, knots and linking number [20] and entanglement [21]. Dynamical systems theory $[22,23]$ provides the critical structures of the vectorlines which may include critical points, limit cycles, invariant tori and invariant sets of dimension $d_{H}>2$. 


\subsection{Surface type structures}

Subsets of the flow field $\mathcal{D} \subset R^{3}$ with Hausdorff dimension $1<d_{H} \leq 2$ are called surface type structures. The vector fields relevant to combustion flows are velocity, vorticity and various flux vector fields. Clearly, subsets corresponding to reaction zones are of particular importance in combustion flows. These reaction zones can be approximated by propagating surfaces [24] if the chemical reactions are sufficiently fast. Propagating surfaces in both reacting and non-reacting turbulent flows may develop singularities in finite time [25] in contrast to materially invariant surfaces. In particular, cusp singularities are an identifying characteristic of propagating surfaces in the spatial description.

\subsection{Volume type structures}

Subsets of the flow field $\mathcal{D} \subset R^{3}$ with Hausdorff dimension $2<d_{H} \leq 3$ are called volume type structures. These structures may have dimension less than three and hence may not fill the Euclidean space $R^{3}$, thus reflecting the intermittent properties of the flow. The Minkowski functional $V_{0}$ is interpreted as the principal measure of the structure, and hence can be computed if the volume type structure is measurable. The functionals $V_{1}, \cdots, V_{3}$ may not exist for structures with a fractal dimension. However, if the dimension of the volume type structure is equal to three and the boundary is sufficiently smooth, then the Minkowski functionals $V_{1}$ to $V_{3}$ are computable and the latter is then the Euler number of the surface.

\section{DNS formulation and datasets}

The analysis is applied to study topologies of flame-flame interactions in highly turbulent premixed flames using the DNS data of Hawkes et al. [26]. The DNS corresponds to a temporally evolving rectangular slot-jet premixed flame in intense shear turbulence. The three-dimensional configuration (see Fig.1) comprises a lean hydrogen-air planar jet of equivalence ratio 0.7 and preheated to $700 \mathrm{~K}$ issuing into a quiescent mixture of adiabatic fully burnt products at the same 
equivalence ratio.

Three simulations with varying jet Damköhler number $\mathrm{Da}_{j} \equiv\left(H / U_{j}\right) /\left(\delta_{L} / s_{L}\right)$ were performed while the jet Reynolds number $\operatorname{Re}_{j} \equiv U_{j} H / v_{u}$ is held constant at a value of 10000 . The simulation domain is rectangular Cartesian and has dimensions of $16 \mathrm{H} \times 20 \mathrm{H} \times 12 \mathrm{H}$ in the streamwise (X), transverse $(\mathrm{Y})$ and spanwise $(\mathrm{Z})$ directions, respectively. The domain boundaries are treated as periodic in the $\mathrm{X}$ and $\mathrm{Z}$ directions, while characteristic outflow boundary conditions are specified at the Y boundaries.

The simulations were performed using the DNS code S3D [27] which solves the compressible Navier-Stokes, continuity, species and energy conservation equations using high order explicit finite difference schemes for spatial $\left(8^{\text {th }}\right.$ order$)$ and temporal $\left(4^{\text {th }}\right.$ order $)$ derivatives. Mixtureaveraged transport coefficients are specified by interfacing with the TRANSPORT library and finite rate chemical kinetics are prescribed using the CHEMKIN library. For the present simulations a 9-species, 19-reaction chemical mechanism was employed [28]. The domain is discretised using a fixed uniform mesh with spacing $18 \mu \mathrm{m}$ in the lowest Damköhler number case (Da-) and $36 \mu \mathrm{m}$ in the highest Damköhler number case ( $\mathrm{Da}+)$ while the time step for advancing the simulation was $2.5 \mathrm{~ns}$ and $5 \mathrm{~ns}$ in the two cases, respectively.

The flame regimes within these data sets are discussed in some detail within the paper of Hawkes et al. [26]. In particular it is noted that broken reaction zones are not observed, and the reaction zones, whilst highly wrinkled, remain intact in the sense that products and reactants are always separated by a strong burning zone. Furthermore transition to a distributed burning regime was not observed and there was little evidence of significant mixing occurring within the reaction zones. Several articles referenced by Hawkes et al. [26] offer explanations for the delay of regime transition until much higher Karlovitz numbers. Hence a flamelet approach based on a reaction progress variable describes the chemical field sufficiently well for the analysis method presented below to be a valid and tractable method to extract flame topological features. 


\section{Formulation of the analysis method}

Assuming surface-type reaction zones, flame-flame interactions can be considered to occur when two flame sheets collide. Here, a flame sheet is defined based upon the isosurface of a reaction progress variable $c$, defined (for example) as a normalised species mass fraction $Y_{\alpha}$ :

$$
c=\frac{Y_{\alpha}-Y_{\alpha R}}{Y_{\alpha P}-Y_{\alpha R}}
$$

where the subscripts $R$ and $P$ denote reactants and products, respectively. At the moment of isosurface collision, there exists no flame normal vector i.e. there is a critical point where the gradient of the progress variable is zero. It is not possible to specify an isosurface value and expect to capture a collision on an instantaneous basis. However, by searching for the critical points within the flow all the collisions or "flame-flame interactions" can be found and the corresponding isosurface values deduced.

The location of each critical point and its associated properties are determined through Newton's method [29] based on a tri-quintic interpolation stencil with global $C^{2}$ continuity. Various other interpolants of higher and lower order were shown to give very high reproducibility indicating that the scalar field is well resolved and that the critical points are physical. Due to the large size of the simulation domain, see section 3, various filtering steps were first used to isolate the regions in which the interpolant was likely to contain a critical point. This was done first through tri-linear interpolation of the eight surrounding nodes to ensure that the interpolant of every component of the scalar field gradient contained a zero-valued isosurface. The approximate solution for the critical point location was then calculated based upon a linearisation of the gradient field around each of the eight nodes. If none of the eight solutions was within the feasible domain, as defined by the eight nodes plus an additional safety halo region, then it was assumed the three zero-valued gradient isosurfaces did not intersect within the cell and a critical point did not exist within it. Otherwise the mean of all feasible solutions was taken as the best estimate of the critical 
point location and provided as input to the Newton's Method algorithm as the first estimate. Examples of the local isosurface topology around two critical points found in this manner are shown in Fig.2.

\subsection{Statistical Variables}

Due to the zero gradient of progress variable at critical points, a Taylor expansion around a critical point reduces to

$$
c(\mathbf{a}+\mathbf{x})=c(\mathbf{a})+\frac{\mathbf{x}^{T}}{2} \underline{\underline{H}}(c(\mathbf{a})) \mathbf{x}+\ldots
$$

such that the Hessian $H(c)$ describes the local field to second order accuracy. The eigenvalues of the Hessian are real, giving the curvature along each of the three orthogonal principal axes. Provided that the orientation is considered to be unimportant, these eigenvalues fully define the local topology to second order. For instance, Fig. 2a shows two positive and one negative eigenvalue and is quite different topologically to the situation shown in Fig.2b with two negative and one positive eigenvalue. Conversion to spherical coordinates proves useful for a more effective description. Equations 4, 5 and 6, where $\lambda_{1}>\lambda_{2}>\lambda_{3}$, give latitude $\phi$ about pole vector $\left[e_{\lambda_{1}}, e_{\lambda_{2}}, e_{\lambda_{3}}\right]$ and longitude $\theta$ about meridian vector $\left[e_{\lambda_{1}}, 0,-e_{\lambda_{3}}\right]$ with $\kappa$ providing the magnitude of the eigenvalues, i.e. an overall measure of curvature. Hence $\theta$ and $\phi$ become shaping parameters and $\kappa$ becomes a scaling parameter with dimensions of length ${ }^{-2}$. The shape factors $\phi$ and $\theta$ are normalised to +/unity. This conversion is shown graphically in Fig.3 highlighting the feasible domain and loci of eigenvalue sign changes.

$$
\begin{aligned}
& \theta=\frac{6}{\pi} \arctan \left(\frac{\left(\lambda_{1}-2 \lambda_{2}+\lambda_{3}\right) / 6^{\frac{1}{2}}}{\left(\lambda_{1}-\lambda_{3}\right) / 2^{\frac{1}{2}}}\right) \\
& \phi=\frac{2}{\pi} \arctan \left(\frac{\left(\lambda_{1}+\lambda_{2}+\lambda_{3}\right) \cos \left(\frac{\theta \pi}{6}\right) / 3^{\frac{1}{2}}}{\left(\lambda_{1}-\lambda_{3}\right) / 2^{\frac{1}{2}}}\right) \\
& \kappa=\left(\lambda_{1}^{2}+\lambda_{2}^{2}+\lambda_{3}^{2}\right)^{\frac{1}{2}}
\end{aligned}
$$


The shape factors define a continuous two-dimensional domain fully describing the range of local topology of flame-flame interactions. Fig. 4 shows the canonical topology corresponding to example pairs of shape factors in a equirectangular projection of $\theta$ and $\phi$ at intervals across their normalised range. Full color is available in the online version of this article. Each drawing uses a colormap ranging from blue for cold (low progress variable) to red for hot (high progress variable). Hence it is possible to infer the direction of propagation of each flame element. On this basis, the topologies appearing on the left of the Figure correspond to outward-propagating spherical flame pockets ("product pocket"), while those on the right are propagating spherically inwards ("reactant pocket"). The more complex topologies appearing towards the bottom right of centre correspond to cylindrical propagation towards a common axis ("tunnel closure", see Fig.2a), while those appearing towards the top left of centre are propagating cylindrically away from a common axis ("tunnel formation", see Fig.2b). The background colours correspond to the signs of the original eigenvalues as shown in Fig.3 i.e. from left to right the sections denote eigenvalue signs of $(---),(--+),(-++)$ and $(+++)$. The lines between each colour indicate the locus of points at which an eigenvalue changes sign. This shape-factor domain combined with the other relevant variables including $\kappa$, progress variable $c$ and reaction rate provide an excellent starting point for gathering statistical data about the flame-flame interactions found within the DNS database. It should be noted that critical points found within regions of the domain with progress variable close to either zero or unity and with very low curvature $\kappa$ have been filtered out.

\section{Results and discussion}

The DNS datasets described in section 3 were analysed using the methods described above. It should be noted that the large size of the datasets resulted in a very large sample of critical points. The spatial datasets for analysis were chosen at an instant in time when the turbulent burning velocity is close to maximum. A suitable progress variable $c$ was defined using the mass fraction of $\mathrm{H}_{2} \mathrm{O}$ for simplicity. It should be noted that the analysis method is fairly insensitive the choice 
of progress variable provided it is continuous and monotonic. Critical points were identified and recorded together with their corresponding shape factors, curvature value, and progress variable value. Histograms showing the frequency of occurrence of all the critical points are shown in Fig. 5 for for the test-cases Da- (left, $\left.\mathrm{Da}_{j}=0.13\right)$ and $\mathrm{Da}+\left(\right.$ right, $\left.\mathrm{Da}_{j}=0.54\right)$. The critical points have been grouped broadly into four distinct types based on the signs of their eigenvalues, and the type of each critical point is indicated by the colour of the bar on the histogram. The interaction types are shown in Fig. 4, examples of the "tunnel formation" and "tunnel closure" events are given in Fig. 2 and all types were explained qualitatively in Section 4.1.

The plots are bimodal, indicating that critical points are most often found for either low or high values of the mean progress variable. The relative lack of interactions in the mid-range of mean progress variable is clear, and indicates that flame-flame interactions are found most often close to either the leading edge or trailing edge of the turbulent flame brush. This is to be expected due to the comparatively large reaction rate in the intermediate range corresponding to a larger rate of change of progress variable and thus a lower probability of observing an interaction in progress from a snapshot of the field at a single instance in time. There are clear differences between the two Damköhler number cases. More interactions are observed in the leading edge of the flame brush (lower progress variable) in the Da- case, and there are more interactions in the middle of the flame brush. By contrast, in the Da+ case, most of the interactions take place towards the trailing edge of the flame brush. The local alignment between the progress variable isosurface normals and the most compressive principal strain rate is observed to increase from the leading to the trailing edge [30]. This, coupled with the less than unity Lewis number associated with lean hydrogen/air mixtures, results in increasing normalized mean conditional scalar dissipation rate and a corresponding decrease of the normalized mean flame thickness. The net effect is to increase the the degree of interaction towards the trailing edge of the flame brush. This also reflects the nature of the local flame structure in each case, with higher Damköhler number indicating a more coherent sheet-like structure, and vice versa. The structures observed are mainly of the 
surface type as introduced in Section 2, although there is a tendency to form line-type structures in limiting cases.

The relative occurrence of each type of interaction is interesting, and is similar for both cases. It is clear that "pocket" events are relatively rare by comparison with the other two types of behaviour, especially over the central portion of the flame brush. The very low occurrence of reactant pockets at all progress variable locations is a consequence of the transient nature of such an event. Surrounded on all sides by hot products, the reactants are rapidly consumed $[9,10]$, making such events difficult to capture in any single snapshot. Product pockets are more frequent towards the trailing edge in both Damköhler number cases. Such events also occur close to the leading edge in the lower Da case, but these are infrequent in the higher Da case. Again this is consistent with the more sheet-like flame structure at high Da.

A somewhat different perspective is provided by the density plot shown in Fig.6. Here, the log frequency of occurrence of each pair of shape factors is indicated by the intensity i.e. light areas indicate fewer interactions. The mean progress variable value at which this flame-flame interaction topology is observed is indicated by the color, which is consistent with Fig.4. Interaction events are strongly clustered along the lines within the space where the eigenvalues change sign. These correspond to interactions which are predominantly two-dimensional such as two ridges meeting or expansion/contraction of a cylindrical tunnel of products/reactants. It is clear that certain topologies are favoured during flame-flame interaction, and these can be deduced by comparing Fig.6 directly with Fig.4. In particular, tunnel formation and tunnel closure are favoured, along with their respective limiting cases of planar interaction either head-on or back-to-back. The colour shows that the topology is related to location within the flame brush. The blue regions near the top right indicate that head-on interactions are favoured towards the reactant side, while the redder regions to the lower left indicate that back-to-back interactions are favoured towards the product side. The differences observed between the two Damköhler number cases are consistent with those observed in Fig.5, i.e. there are more interactions at higher values of mean progress 
variable in the higher Da case, and vice versa.

Further and more detailed trend analysis is ongoing, including factors such as scale, reaction rate, and local hydrodynamic strain rate. Work is also proceeding to investigate how the observed topological behaviour might be incorporated in submodels of the FSD and G-equation approaches. Clearly there is potential for significant enhancement of model terms describing flame surface area creation and destruction through propagation and curvature.

\section{Conclusions}

The topology of turbulent premixed flames has been analysed using a DNS dataset of interacting turbulent hydrogen-air flames. Starting from a general approach to evaluating the topology of line, surface and volume fields, a method based on the eigenvalues of the scalar Hessian has been used to find the critical points of a field of reaction progress variable based on $\mathrm{H}_{2} \mathrm{O}$ mass fraction, as obtained from the DNS dataset. The full set of possible flame-flame interaction topologies has been identified, and these have been parametrised using two shape factors derived from the eigenvalue analysis. The relative frequency of occurrence of all the types of interaction topology has been extracted from the DNS data. It has been found that essentially cylindrical topologies corresponding to either tunnel closure or tunnel formation are favoured over topologies that are more spherical in nature. Differences are observed between the interaction behaviour at two different Damköhler numbers, reflecting the respective differences in the local flame structure. There is little interaction in the middle of the turbulent flame brush, and most interactions involve either head-on or back-to-back configurations occurring respectively at the leading or trailing edge of the flame brush.

\section{Acknowledgements}

This paper has been developed as a result of a mobility stay funded by the Erasmus Mundus Programme of the European Commission under the Transatlantic Partnership for Excellence in 
Engineering - TEE Project.

This research used resources of the National Center for Computational Sciences at Oak Ridge National Laboratory, which is supported by the office of Science of the US Department of Energy under contract DE- AC05-00OR22725. The work at Sandia National Laboratories was supported by the Division of Chemical Sciences, Geosciences, and Biosciences, Office of Basic Energy Sciences of the US Department of Energy and by the US Department of Energy SciDAC Program.

[1] N. Chakraborty, R.S. Cant, Combust. Flame 156 (2009) 1427-1444.

[2] D.O. Lignell, J.H. Chen, H.A. Schmutz, Combust. Flame 158 (2011) 949-963.

[3] H. Kolla, R.W. Grout, A. Gruber, J.H. Chen, Combust. Flame 159 (2012) 2755-2766.

[4] R.S. Cant, S.B. Pope, K.N.C. Bray, Proc. Combust. Inst. 23 (1990) 809-815.

[5] N. Peters, Turbulent Combustion, Cambridge University Press, Cambridge, U.K., 2000.

[6] C. Pantano, D.I. Pullin, Combust. Flame 137 (2004) 295-305.

[7] E.R. Hawkes, R.S. Cant, Proc. Combust. Inst. 28 (2000), 51-58.

[8] T.D. Dunstan, N. Swaminathan, K.N.C. Bray, J. Fluid Mech. 709 (2012) 191-222.

[9] W. Kollmann, J.H. Chen, Proc. Combust. Inst. 27 (1998) 927-934.

[10] J.H. Chen, T. Echekki, W. Kollmann, Combust. Flame. 116 (1999) 15-48.

[11] M. Yoda, L. Hesselink, M.G. Mungal, J. Fluid Mech. 279 (1994) 313-350.

[12] L. Wang, N. Peters, J. Fluid Mech. 554 (2006) 457-475.

[13] Y. Shim, S. Tanaka, M. Tanahashi, T. Miyauchi, Proc. Combust. Inst. 33 (2011) 1455-1462.

[14] Y. Minamoto, N. Swaminathan, R.S. Cant, T. Leung, "Reaction zones and their structure in MILD combustion", Combust. Sci. Tech., in press, 2013.

[15] W. Kollmann, Fluid Mechanics in Spatial and Material Description, University Readers, San Diego, 2010.

[16] V.I. Bogachev, Measure Theory, vol.1, Springer Verlag, New York, 2000.

[17] H. Minkowski, Math. Ann. 57 (1903) 447-495.

[18] H. Federer, Geometric Measure Theory, Springer Verlag, Berlin, 1969.

[19] Y.B. Pesin, Dimension Theory in Dynamical Systems, The University of Chicago Press, Chicago, 1997.

[20] W. Fulton, Algebraic Topology, Springer Verlag, New York, 1995.

[21] C.F. Barenghi, R.L. Ricca, D.C. Samuels, Physica D 157 (2001) 197-206.

[22] B. Hasselblatt, A. Katok, A First Course in Dynamics with a Panorama of Recent Developments, Cambridge University Press, Cambridge, U.K., 2003. 
[23] S. Wiggins, Introduction to Applied Nonlinear Dynamical Systems and Chaos, Springer Verlag, New York, 2003.

[24] S.M. Candel, T.J. Poinsot, Combust. Sci. Tech. 70 (1990) 1-15.

[25] S.S. Girimaji, S.B. Pope, J. Fluid Mech. 234 (1992) 247-277.

[26] E.R. Hawkes, O. Chatakonda, H. Kolla, A.R. Kerstein, J.H. Chen, Combust. Flame 159 (2012) 2690-2703.

[27] J.H. Chen, A. Choudhary, B. de Supinski, M. DeVries, E.R. Hawkes, S. Klasky, W.K. Liao, K.L. Ma, J. MellorCrummey, N. Podhorszki, R. Sankaran, S. Shende, C.S. Yoo, Comput. Sci. Discovery 2 (2009) 015001.

[28] J. Li, Z. Zhao, A. Kazarov, F.L. Dryer, Int. J. Chem. Kinet. 36 (2004) 566-575.

[29] W.H. Press, S.A. Teukolsky, W.T. Vetterling, B.P. Flannery, Numerical Recipes, 3rd ed., Cambridge University Press, Cambridge, U.K., 2007.

[30] S. Chaudhuri, H. Kolla, E.R. Hawkes, J.H. Chen, C.K. Law, "Flame Thickness and Conditional Scalar Dissipation Rate in a Premixed Temporal Turbulent Reacting Jet”, J. Fluid Mech. (2013), submitted. 


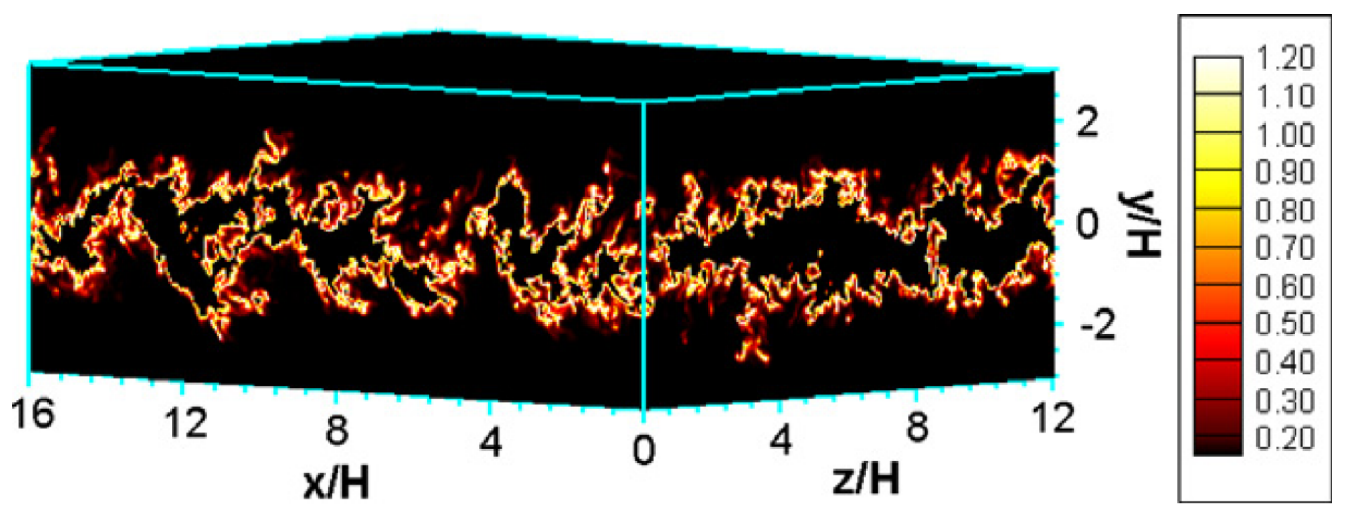

Figure 1: Reaction rate from DNS data of Hawkes et al. [26].

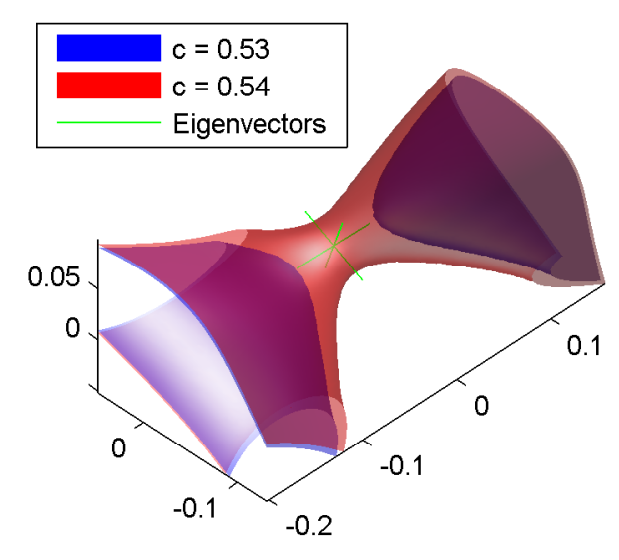

(a) Tunnel Closure

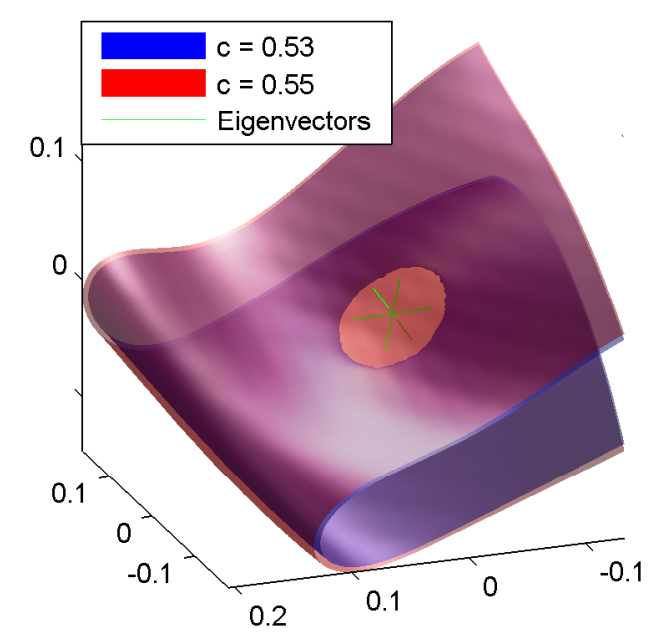

(b) Tunnel Formation

Figure 2: Examples of flame-flame interactions taken from DNS data. All units in mm 

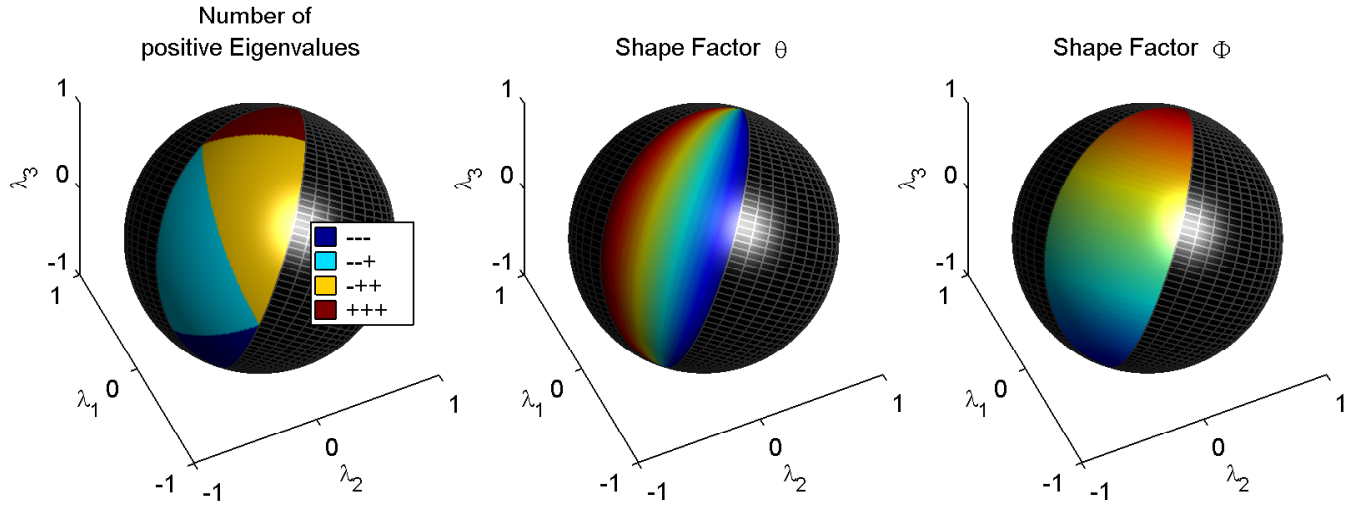

Figure 3: Feasible domain of $H(c)$ eigenvalues correlating to shape factors $\theta$ and $\phi$ as defined in equations 4 and 5 .

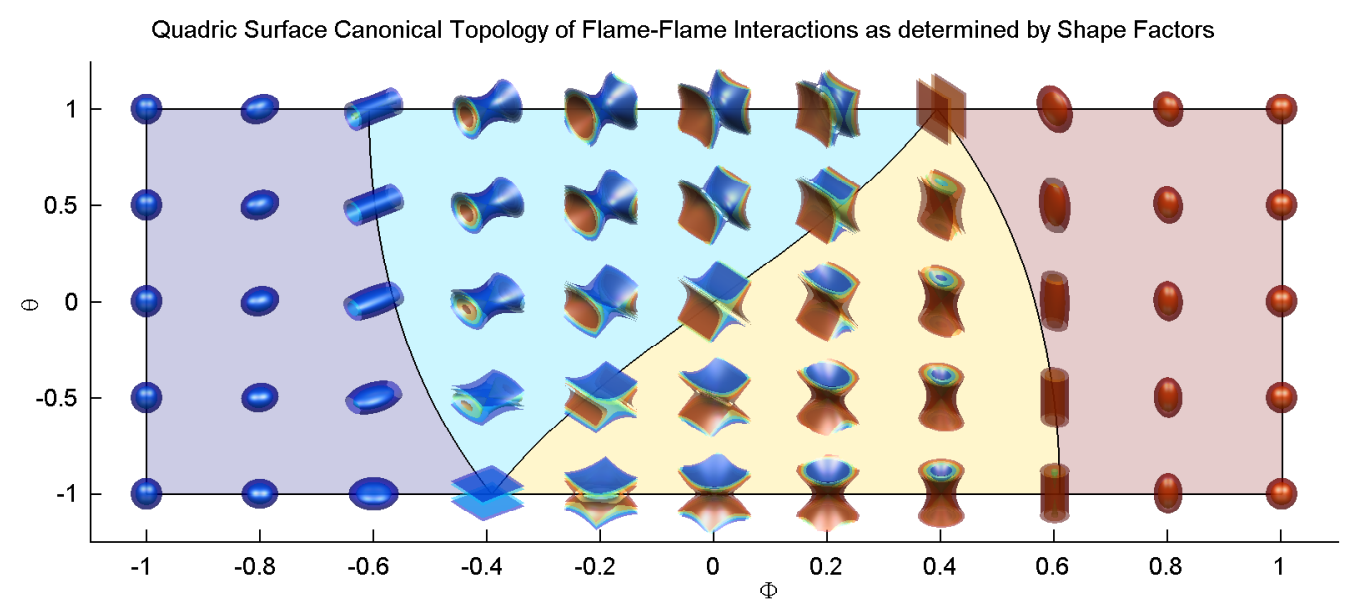

Figure 4: Quadric surface canonical topology of flame-flame interactions as determined by the shape factors. The boundaries between background area denote a change in the sign of an eigenvalue. 


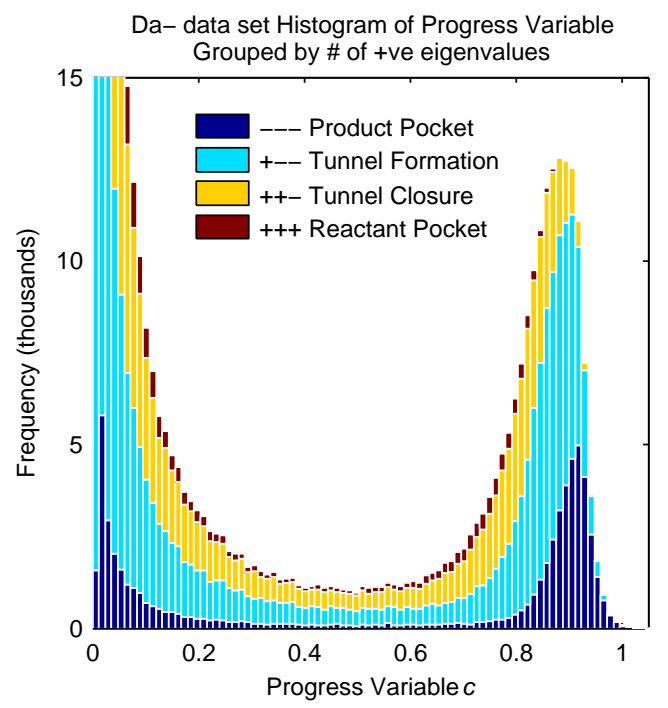

(a) Lower Da number test-case

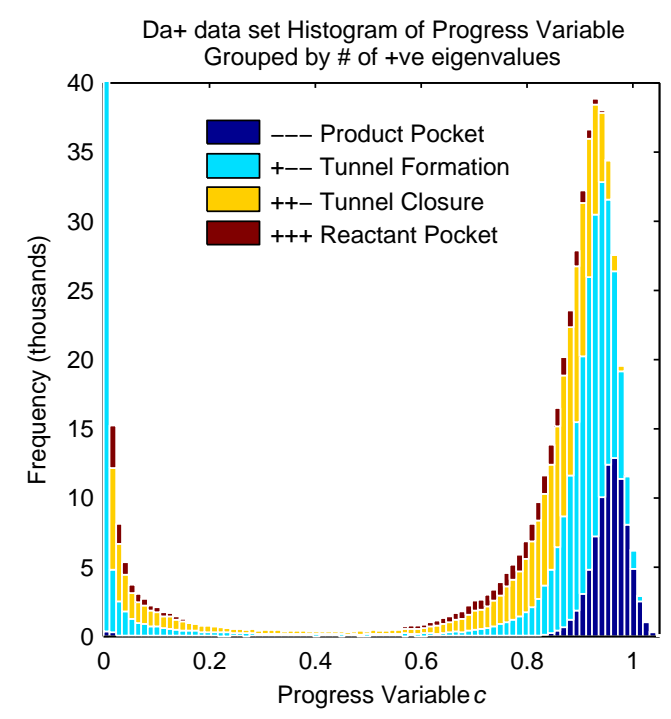

(b) Higher Da number test-case

Figure 5: 1D Histogram of progress variable at critical points with coloured stacks denoting sign of the eigenvalues.

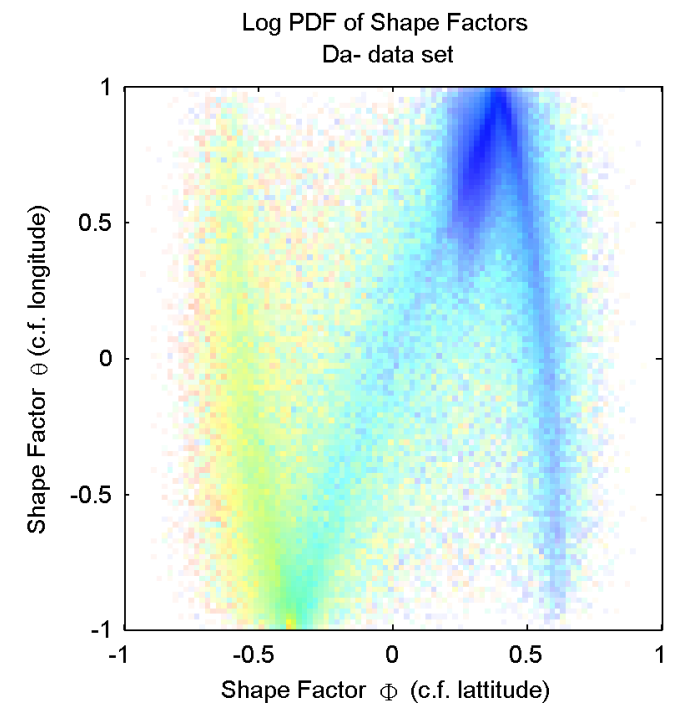

(a) Lower Da number test-case

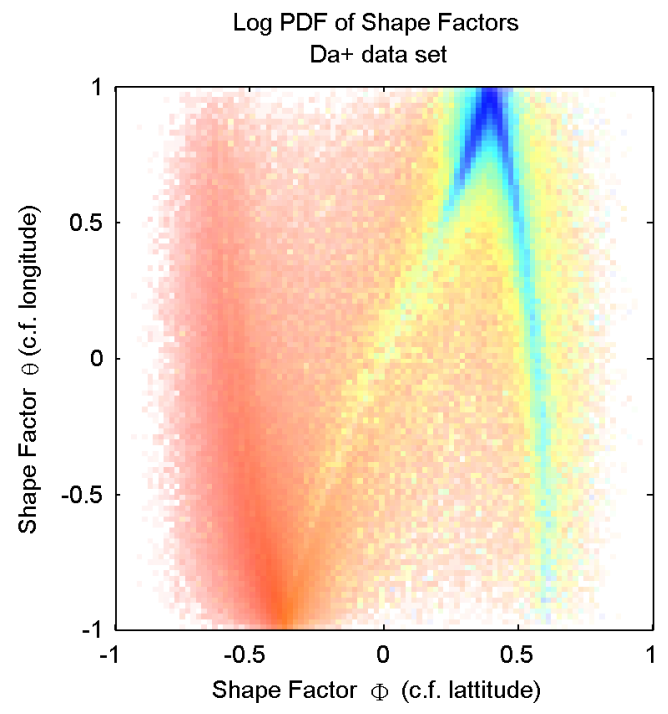

(b) Higher Da number test-case

Figure 6: Log histogram on $\phi-\theta$ domain. Colour denotes mean progress variable $c$. 